\title{
Review: Studying Physics, getting to know Python: RC circuit, simple experiments and coding with Raspberry Pi
}

\author{
Dirk Colbry ${ }^{1}$ \\ ${ }^{1}$ Michigan State University
}

June 25, 2020

The article "Studying Physics, getting to know Python: RC circuit, simple experiments and coding with Raspberry P $i$ " introduces a hands-on, integrative studies approach to teaching electronics, physics, and computer science. I believe it provides all of the steps (and code) needed to reproduce the basic exercises in the classroom and there is room to add on new ideas. The course is designed to use Raspberry Pis; because of the affordable nature of the equipment (less than a textbook). I could see proposing to use this setup as an online lab where students purchase the equipment and run the lab while they are quarantined at home.

Unfortunately, as written the article is over 8,0000 words when counting figures which is significantly over the 3000 word limit given in the CISE guidelines for department papers:

Up to 3000 words in length, including the abstract, references, bios, figures (see below), and all other text in the article. When counting words, note that tables and figures should be counted as 250 words each.

The article is over 8,000 words when counting the figures and I would be concerned that cutting it to 3000 words would take away from one of the article's strengths. A compromise would be to provide the examples in an online git repository and reference the repository in the article.

For example, a GitHub repository could be created for the "supplementary materials" of code and figures, with a description in the README, and an appropriate educational materials public license (ex. CC-BY for content and BSD-3 for code). We could also host the documents to the Github.org CISE-Ed group, but that would be optional. What we would expect is that no matter where the repository is located it should also be archived in Zenodo to get a permanent identifier. Then, the Zenodo archive DOI can easily be cited in the paper.

Although using Raspberry Pis in the classroom is not a new concept, this paper does a good job presenting the elegance of their use in an integrated studies course. As I was reading though the description, it occurred to me that an Arduino has a built-in A/D converter and may simplify the circuit connections. However, I now realize that using an Arduino would require a lot more software and setup on a dedicated computer (laptop or desktop) to analyze the data. Thus, the Raspberry Pi is an elegant and affordable almost-in-one solution (it still requires the A/D converter, circuit components, keyboard, monitor, mouse, etc.).

The first experiment (turning on and off an LED) is a great place to start. Students get immediate feedback to see how a few lines of code can impact something in the "real world." I assume that instructors would mention that if we can turn on an LED, we could turn on a variety of devices (such as a relay to turn on a fan or a coffee maker). Although the primary learning goal is to understand resistors, capacitors and circuits, I think connecting what they are doing to engineering is a powerful motivator for many students. I like that that this simple and easy project could truly spark creativity in the students. 
Because most of the computation is hidden away inside of the libraries, the resulting code is extremely easy to read and so short that it can be included in the paper. As someone who teaches Python and is familiar with circuits, I can immediately see the benefit of this approach. Even for novice students, I do not think that learning the coding parts will overshadow the primary learning goals. In fact, I agree with the authors that the integrated nature of the assignments would be fun and extremely motivational as compared to a similar lab with expensive lab equipment.

One thing missing from the paper is any sort of evaluation of the success of the approach. At the very least, it would be nice to have some quotes from students (both good and bad) describing how this approach landed in the classroom. Even better would be some measure of student learning, with an ideal measure compared to a more traditional classroom. I realize setting up a classroom experiment is difficult (and probably outside the scope of this article); however, having tangible data could really help sell this approach for adoption in other classrooms.

Although I think it would take a lot of work to get right and scale, I really want someone to try this technique with remote learners. Students could purchase a Raspberry Pi kit for less than the cost of a textbook $(<\$ 100)$ and set up a lab in their own home. This would add a huge layer of complexity as students, would then need to install the OS and figure out how to connect things on their own. However, the nice thing about this setup is that it could work well in our current remote-learning world. As soon as this article comes out I plan to share it with a colleague who has been teaching basic circuits, to see if this approach would work in their classroom.

Here are a few other minor suggestions. These do not necessarily directly align with the learning goals, but may be worth looking into:

- The paper (or supplementary materials) should include a parts list (with approximate prices) to demonstrate the cost effectiveness of this approach to readers new to the technology.

- It would be nice to connect the A/D converter to some sort of simple resistance-based sensor (e.g., thermocouple). Again, the goal would be to spark creativity in the students by showing them things that connect to real world problems.

- I was also hoping for a final experiment where the changing voltage would be applied to something other than the Raspberry Pi. Maybe some sort of speaker or a fan. Some way for the students to see how their circuit could be used and completely disconnecting it from the digital technology.

- I found a Raspberry Pi GPIO emulator. Although not as good as the real thing, I wonder if something like this could be set up for students and/or instructors to practice on their own computers?

In summary, a good paper that will spark creativity in instructors as well as the students. 